\title{
On the Reduction of Ferrers' Associated Legendre Function.
}

\author{
By Hrishikesh Sircar.
}

(Received 18th January 1929. Received in revised form 11th April 1929.)

Introduction. In the present paper a formula will be obtained to express a Ferrers' Associated Legendre Function of any integral degree and order as a sum of a finite number of Associated Legendre Functions of an order reduced by an even number. When the order is reduced by unity, an infinite series of the functions of reduced order is required. Thus a Ferrers' function can be expressed as the sum of a finite or infinite number of zonal harmonics according as the order of the function is even or odd.

We shall use the notation $T_{n}{ }^{m}(x)$ to represent a Ferrers' Associated Function of degree $n$ and order $m$, while, for brevity, $P^{\prime}{ }_{n}$ and $P_{n}\langle s-1)$ will be taken to denote $\frac{d}{d x} P_{n}(x)$ and $\frac{d^{s-1}}{d x^{s-1}} P_{n}(x)$ respectively

$\S 1$. We have

$$
T_{n} s(x)=\left(1-x^{2}\right)^{\frac{s}{2}-k}\left(1-x^{2}\right)^{k} P_{n}^{(s)}
$$

where $k$ is a positive integer.

Now differentiate Legendre's equation

$$
\frac{d}{d x}\left[\left(1-x^{2}\right) P_{n}^{\prime}\right]+n(n+1) P_{n}=0
$$

$(s-2)$ times and get

$\left(1-x^{2}\right) P_{n}^{(s)}-2(s-1) x P_{n}^{(s-1)}+(n-s+2)(n+s-1) P_{n}^{(s-2)}=0 \ldots$ (i)

Again, differentiate

$$
(n+1) P_{n+1}-(2 n+1) x P_{n}+n P_{n-1}=0
$$

$(s-1)$ times, and

$$
P_{n+1}^{\prime}-P_{n-1}^{\prime}=(2 n+1) P_{n}
$$

$(s-2)$ times, and get

$(n+1) P_{n+1}^{(s-1)}=(2 n+1)\left[x P_{n}^{(s-1)}+(s-1) P_{n}^{(s-2)}\right]-n P_{n-1}^{(s-1)}$

$(2 n+1) P_{n}^{(s-2)}=P_{n+1}^{(s-1)}-P_{n-1}^{(s-1)}$

Adding $(s-1)$ times the latter to the former we have

$(n-s+2) P_{n+1}^{(s-1)}=(2 n+1) x P_{n}^{(s-1)}-(n+s-1) P_{n-1}^{(s-1)}$. 
Hence, from (i), (iv), and (v) we get

$$
\begin{aligned}
\left(1-x^{2}\right) P_{n}^{(s)}=-\frac{(n-s+2)}{2 n+1} \frac{(n-s+1)}{-1} P_{n+1}^{(s-1)} & \\
& \quad+\frac{(n+s)(n+s-1)}{2 n+1} P_{n-1}^{(s-1)} \ldots(\beta)
\end{aligned}
$$

Now multiply by $\left(1-x^{2}\right)$ and apply $(\beta)$ to the right hand side; then,

$$
\begin{aligned}
& \left(1-x^{2}\right)^{2} P_{n}^{(s)}=\frac{(n-s+4) !}{(n-s) !} \frac{1}{(2 n+3)(2 n+1)} P_{n \div-2}^{(s-2)} \\
& -2 \frac{(n+s)(n+s-1)(n-s+1)(n-s+2)}{(2 n+3)(2 n-1)} P_{n}^{(s-2)} \\
& +\frac{(n+s) !}{(n+s-4) !} \frac{1}{(2 n+1)(m-1)} P_{n-2}^{(s-2)} .
\end{aligned}
$$

From this the law can be deduced; the general term in the expression for $\left(1-x^{2}\right)^{k} P_{n}^{(s)}(x)$ is $\quad K_{n, s, k, r} P_{n+k-2 r}^{(s-k)}$, where $K_{n, s, k, r}=(-1)^{k+r}{ }^{k} C_{r} \frac{(n-s+2 k-2 r) !}{(n+s-2 r) !} \frac{(n+s) !}{(n-s) !} 2^{k}$

$$
\times \frac{(2 n-2 r) !(n-r+k) !}{(n-r) !(2 n+2 k-2 r+1) !}(2 n+2 k-4 r+1)
$$

$\S 2$. The desired reduction can now be effected, since, from (a) and $(\gamma)$,

$$
T_{n}^{s}(x)=\left(1-x^{2}\right)^{\frac{s-2 k}{2}} \sum_{r=0}^{k} K_{n, s, k, r} \frac{d^{s-2 k}}{d x^{s-2 k}} P_{n+k-2 r}^{(k)} ;
$$

and, since

$$
\begin{aligned}
& P_{n+k-2 r}^{(h)}=\sum_{p}^{k+p-1} C_{p} \frac{1.3 .5 \ldots(2 n+2 k-4 r-2 p-1)}{1.3 .5 \ldots \ldots(2 n-4 r-2 p+1)} \\
& \times(2 n-4 r-4 p+1) P_{n-2 r-2 p},
\end{aligned}
$$

it follows that

$$
\begin{aligned}
T_{n} s(x)=\sum_{r=0}^{k} K_{n, s, k, r} \sum_{p}^{k+p-1} C_{p} \frac{1.3 .5 \ldots(2 n+2 k-4 r-2 p-1)}{1.3 .5 \ldots(2 n-4 r-2 p+1)} \\
\quad \times(2 n-4 r-4 p+1) T_{n-2 r-2 p}^{s-2 k}(x) \quad \ldots \ldots \ldots
\end{aligned}
$$

The result corresponding to $k=1$ was obtained by Adams by a different method. ${ }^{1}$ The observation made in the introduction is, from $(\kappa)$, quite clear and requires no further comment.

1 Collected Scientific Pupers, 2, 412. Cf. also 2, 376. 
§3. In conclusion, I propose to give an outline of a proof that if the order of any Ferrers' associated Legendre function be reduced by unity, the associated function can be expressed by a series, composed of an infinite number of terms, of associated functions of the reduced order. If we consider $T_{i}{ }^{m+1}(x)$, then it can be written in the form

$$
T_{n}{ }^{m+1}(x)=\left(1-x^{2}\right)^{\frac{1}{2}} \cdot\left(1-x^{2}\right)^{\frac{n}{2}} \cdot \frac{d^{m}}{d x^{m}} P^{\prime}(x) .
$$

Introducing the well-known expression of $P_{n}^{\prime}(x)$ in terms of zonal harmonics and using 1

$$
\sqrt{1-x^{2}}=\frac{\pi}{2}\left[\frac{1}{2}-5\left(\frac{1}{2}\right)^{2} \cdot \frac{1}{4} \cdot P_{2}(x)-9\left(\frac{1}{2.4}\right)^{2} \cdot \frac{3}{6} P_{4}(x) \ldots\right],
$$

$(\delta)$ can be expressed as the product of a series of zonal harmonics with another series of associated functions. This involves products of zonal harmonics and Ferrers' associated Legendre functions; all such products can be converted into series of the same order and of different degrees by means of a formula due to Adams ${ }^{2}$, viz.,

$$
\begin{aligned}
T_{n}{ }^{m}(x) & T_{q}{ }^{p}(x)=\sum_{r}(2 n+2 q-4 r+1) T_{n+q-2 r}^{m+p}(x) \cdot(-)^{r} \\
& \times \frac{(n+q-r) !(2 q-2 r) !(n+m) !}{r !(q-r) !(n-r) !(2 n+2 q-2 r+1) !} \\
& \times\left[\sum_{s=0}^{q-p}(-)^{s} \frac{(q+p+s) !(2 n-2 r+q-p-s) !}{s !(q-p-s) !(q+p-2 r+s) !(n+m-s) !}\right],
\end{aligned}
$$

and the conclusion follows at once.

1 'Todhunter. The functions of Laplace, Lamé and Bessel, p. 114. Also Crelle, 56.

2 Adams. Collected Scientific Papers, 2, 395, the quotation is given in an altered form. There is also another equivalent expression of order $n-p$. 